\title{
Dairy consumption and circulating levels of inflammatory markers among Iranian women
}

\author{
Ahmad Esmaillzadeh ${ }^{1,2, *}$ and Leila Azadbakht ${ }^{1,2}$ \\ 'Department of Nutrition, School of Public Health, Isfahan University of Medical Sciences, PO Box 81745, \\ Isfahan, Iran: ${ }^{2}$ Food Security and Nutrition Research Center, Isfahan University of Medical Sciences, \\ Isfahan, Iran
}

Submitted 13 March 2008: Accepted 20 October 2009: First published online 15 December 2009

\begin{abstract}
Objective: Although recent studies have shown an inverse relationship between dairy consumption and metabolic abnormalities, we are aware of no study evaluating the association between dairy consumption and circulating levels of inflammatory markers. The current study was undertaken to assess the association between the consumption of high-fat and low-fat dairy products and circulating levels of inflammatory markers among Tehrani women aged 40-60 years.

Design: In a cross-sectional study of 486 apparently healthy women aged 40-60 years, we assessed usual dietary intakes by means of an FFQ. Anthropometric measurements were made and fasting blood samples were taken for measuring inflammatory markers.

Results: The reported mean (SD) daily intake of low- and high-fat dairy consumption was 85 (sD 23) and 101 (SD 29) g/d, respectively. After control for age, BMI, waist circumference and other potential confounders, low-fat dairy consumption was inversely associated with C-reactive protein $(\beta=-0.04)$, IL-6 $(\beta=-0.02)$ and soluble vascular cell adhesion molecule-1 $(\beta=-0.06)$; with further adjustment for dietary intakes, the associations remained significant just for soluble vascular cell adhesion molecule-1 $(\beta=-0.03)$. High-fat dairy intake was positively associated with log-transformed values of serum amyloid A $(\beta=0.08)$ and soluble vascular cell adhesion molecule- $1(\beta=0.05)$, both before and after adjustment for all potential confounding variables. No overall significant associations were found between total dairy consumption and inflammation.

Conclusions: The current study indicates an independent relationship between high-fat as well as low-fat dairy consumption, not total dairy intake, and some markers of inflammation and endothelial dysfunction. Further studies are required to identify responsible components of dairy products and related mechanisms of action.
\end{abstract}

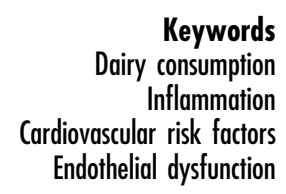

Keywords

airy consumption

lar risk factors

Endothelial dysfunction
Inflammation has been postulated to have a pivotal role in the pathogenesis of many chronic conditions, such as obesity, CVD, diabetes and metabolic syndrome ${ }^{(1-5)}$. Harvard investigators $^{(6)}$ have introduced inflammation as a key driver of these chronic conditions. Circulating levels of inflammatory markers such as C-reactive protein (CRP) and TNF- $\alpha$ are considered to be involved in the development of ischaemic events $^{(7,8)}$. Moreover, recent evidence supports the association between inflammation and endothelial dysfunction ${ }^{(9)}$. Overall, these data indicate that identifying determinants of markers of systemic inflammation and endothelial dysfunction is of great importance in the field of cardiology.

The exact underlying factors of elevated circulating levels of inflammatory markers remain to be identified. Although recent studies have reported obesity ${ }^{(10)}$, smoking ${ }^{(11)}$, hypercholesterolaemia $^{(12)}$ and physical inactivity ${ }^{(13)}$ as major predictors of inflammation, data on dietary determinants of circulating levels of inflammatory biomarkers are scarce. Moreover, limited data available in this field have mainly focused on nutrients ${ }^{(14-17)}$ or dietary patterns ${ }^{(18-20)}$, and little attention has been given to foods, particularly dairy products. Some investigations have reported the relationship between consumption of vegetable oils ${ }^{(21)}$, red meat ${ }^{(22)}$, fruits and vegetables ${ }^{(23,24)}$ and soya ${ }^{(25)}$ with inflammatory biomarkers, but we are aware of no study evaluating the association between dairy consumption and circulating levels of inflammatory markers. On the other hand, recent studies have shown an inverse relationship between dairy consumption and metabolic abnormalities, such as obesity ${ }^{(26-28)}$, diabetes ${ }^{(29,30)}$, metabolic syndrome $^{(31,32)}$ and insulin resistance ${ }^{(33)}$, but the mechanisms have not been fully understood. Dairy products are a rich 
source of $\mathrm{Ca}$; a nutrient seems to be responsible for dairy's beneficial effect ${ }^{(34)}$. Supplementation with $\mathrm{Ca}$ and vitamin $\mathrm{D}$, two major ingredients of dairy products, for 3 years could affect glycaemia and insulin resistance, but not inflammatory markers, among older adults ${ }^{(35)}$. Besides $\mathrm{Ca}$, dairy products contain other nutrients, such as conjugated linoleic acid (CLA), riboflavin and high-quality proteins, that may affect systemic inflammatory levels ${ }^{(36,37)}$. The current study was, therefore, undertaken to assess the association between consumption of high- and low-fat dairy products and circulating levels of inflammatory markers among female teachers aged 40-60 years living in Tehran.

\section{Subjects and methods}

\section{Subjects}

The current cross-sectional study was conducted among a representative sample of female teachers aged 40-60 years living in Tehran selected by a multistage cluster random sampling method. A random sample of 583 female teachers were invited to participate in the current study and 521 women agreed to do so. Participants with a prior history of CVD, diabetes, cancer and stroke were excluded because of possible changes in diet. We also excluded those with possible inflammation. We also excluded subjects who had left $>70$ items blank on the FFQ, who reported a total daily energy intake outside the range of $3347 \cdot 2-17522 \cdot 8 \mathrm{~kJ}(800-4200 \mathrm{kcal})$ and those taking medications that would affect serum lipoprotein, blood pressure and carbohydrate metabolism. These exclusions resulted in 486 subjects for present analysis. All participants provided an informed written consent.

\section{Assessment of dietary intake}

Usual dietary intake was assessed using a validated 168-item semi-quantitative $\mathrm{FFQ}^{(28,31)}$. Briefly, our validation study included randomly chosen participants of 132 subjects (not included in the current study) by comparing nutrient consumption determined using responses to the FFQ on two occasions 1 year apart. Comparative validity was determined by comparison with intake estimated from the average of twelve $24 \mathrm{~h}$ dietary recalls (one for each month of the year). The findings from the current validation study can be found elsewhere ${ }^{(28,31)}$. We concluded from the validation study that the FFQ provides reasonably valid and reliable measures of the average long-term dairy intake.

All the questionnaires were administered by a trained dietitian. The FFQ consisted of a list of foods with standard serving sizes commonly consumed by Iranians. Participants were asked to report their frequency of consumption of a given serving of each food item during the previous year on a daily (e.g. bread), weekly (e.g. rice and meat) or monthly (e.g. fish) basis. The reported frequency for each food item was then converted to a daily intake. Portion sizes of consumed foods were converted to grams using household measures. Total energy intake was calculated by summing up energy intakes from all foods ${ }^{(38)}$.

We considered low-fat $(<2 \%)$ dairy as skim or low-fat milk and low-fat yoghurt. High-fat dairy ( $\geq 2 \%$ ) was considered as high-fat milk, whole milk, chocolate milk, cream, high-fat yoghurt, cream yoghurt, cream cheese, other cheeses and ice cream. Before performing separate analysis for lowand high-fat dairy, we considered the total dairy intake as an independent variable. But, we did not reach significant finding for any of the inflammatory markers. So, decided to separately analyse for low- and high-fat dairy intake.

\section{Assessment of biomarkers}

Detailed information about the measurement of inflammatory biomarkers could be found elsewhere ${ }^{(18)}$. Briefly, a blood sample was drawn between 07.00 and $09.00 \mathrm{~h}$ into Vacutainer tubes from all study participants after $>12 \mathrm{~h}$ overnight fasting. Blood samples were centrifuged within $30-45 \mathrm{~min}$ of collection, and plasma was frozen at $-70^{\circ} \mathrm{C}$ until analysis. CRP concentrations were measured by using an ultrasensitive latex-enhanced immunoturbidimetric assay (Randox Laboratories Ltd, Crumlin, UK). Circulating levels of serum amyloid A (SAA), E-selectin, soluble intercellular adhesion molecule-1 (sICAM-1) and soluble vascular adhesion molecule-1 (sVCAM-1) were measured by commercially available ELISA and standards (Biosource International Ltd, Camarillo, CA, USA and Bender Med Ltd, Vienna, Austria). TNF- $\alpha$ and IL- 6 were assayed by enzymelinked immunoassay assay (Bender Medsystem kits). Interand intraassay CV for all markers were $<10 \%$. Blood lipid levels were assessed according to the standard methods ${ }^{(39)}$.

\section{Assessment of otber variables}

Weight and height were measured, and BMI was calculated accordingly ${ }^{(39)}$. Waist circumference (WC) was measured at the narrowest level over light clothing using an unstretched tape measure. Data on family history of diabetes were collected as the participants' oral responses to the pre-tested questionnaire. The criterion for family history of diabetes was having at least one first-degree relative with a diagnosis of diabetes after 30 years of age. Data on physical activity were obtained using the subjects' oral responses to a pre-tested questionnaire and expressed as metabolic equivalent hours per week $(\text { MET } \times \mathrm{h} / \text { week })^{(18)}$. Additional covariate information regarding age, smoking habits, menopausal status, medical history and current use of medications was obtained using validated questionnaires. Participants' blood pressure was also assessed according to a standard protocol $^{(40)}$.

\section{Statistical methods}

Cut-off points for quintiles of high- and low-fat dairy intakes were calculated and subjects were categorised based on quintile cut-off points. Significant differences in general 
characteristics across quintiles of high- and low-fat dairy intakes were assessed using a one-way ANOVA with Tukey post hoc test. Chi-square test was used to detect significant differences in the distribution of subjects across quintiles of high- and low-fat dairy intakes with regard to categorical variables. We determined age- and energy-adjusted means for dietary variables across quintiles of high- and low-fat dairy intakes by using a general linear model. Analysis of covariance was used to compare these means.

Before statistical analyses, we looked at outliers in each variable and excluded them from the final analysis. The distribution of inflammatory markers was highly skewed. Therefore, logarithmically transformed values of these markers were used in all analyses. Geometric means of inflammatory markers across quintiles of high- and lowfat dairy intakes were computed by using analysis of covariance in three different models. In the first model, we adjusted for age (continuous), BMI (continuous) and WC (continuous). In the second model, we additionally adjusted for smoking (yes or no), physical activity (continuous), total energy intake (continuous), use of oestrogen (yes or no), menopausal status (yes or no) and family history of diabetes or stroke (yes or no), systolic and diastolic blood pressure (continuous), fasting plasma glucose, serum TAG concentrations, total cholesterol and HDL and LDL cholesterol. Finally, we added dietary variables into the model including cholesterol intake, consumption of meats and fish, fruit and vegetables, whole and refined grains, hydrogenated and nonhydrogenated vegetable oils, percentage of energy from fat and mutual effects of high- and low-fat dairy intakes (all as continuous). Analysis of covariance was used for comparison of inflammatory markers across quintiles.

To determine the association of high- and low-fat dairy consumption with inflammatory markers, we used multiple linear regression analysis. In these models, log-transformed plasma concentrations of inflammatory markers were used to achieve normal distributions. We looked at the mentioned associations in three different models with covariates as those used in the above-mentioned models. The Statistical Package for Social Science statistical software package version 9.05 (SPSS Inc., Chicago, IL, USA) was used for all statistical analyses.

\section{Results}

The reported mean (SD) daily intake of low- and high-fat dairy consumption was 85 (SD 23) and 101 (SD 29) g/d, respectively. The food items that contributed most to low-fat dairy intakes were low-fat yoghurt and those that contributed most to high-fat dairy intake were high-fat yoghurt and cheeses.

Participants' general characteristics and dietary intakes across quintiles of dairy consumption are shown in Table 1. Compared to participants in the lowest quintile, those in the upper quintile of low-fat dairy consumption had lower BMI and WC, were older, more physically active and more likely to be current oestrogen users. Individuals in the top quintile of high-fat dairy consumption had lower means of BMI and WC, were older, and more likely to be current oestrogen users than those in the third quintile. No significant difference was seen regarding the distribution of current smokers and those with family history of diabetes or stroke across quintiles of either lowor high-fat dairy consumption. Higher intakes of lowfat dairy were associated with a healthier diet; those subjects in the upper category consumed more fruit, vegetables, whole grains, non-hydrogenated vegetable oils, dietary fibre, vitamin $\mathrm{B}_{2}, \mathrm{Mg}$ and $\mathrm{Ca}$ and less hydrogenated vegetable oils, high-fat dairy and energy from fat. Individuals in the top category of high-fat dairy intakes consumed more fruit, hydrogenated vegetable oils, cholesterol, vitamin $\mathrm{B}_{2}$, Ca and energy from fat and less whole grains, refined grains, low-fat dairy, non-hydrogenated vegetable oils, $\mathrm{Mg}$ and energy from carbohydrate.

Geometric means of circulating inflammatory marker levels across quintile categories of dairy consumption are given in Table 2. Compared to those in the lowest quintile, individuals in the top quintile of low-fat dairy consumption had lower circulating levels of CRP, TNF- $\alpha$, IL-6, E-selectin, sICAM-1 and sVCAM-1. Adjustment for potential confounding variables attenuated these associations. However, after further control for dietary intakes, a significant association between low-fat dairy consumption and circulating levels of IL- 6 and sVCAM- 1 was found; those in the highest category had lower circulating levels of these markers. There was a significant association between high-fat dairy consumption and serum levels of TNF- $\alpha$, SAA, E-selectin and sVCAM-1. Adjustment for age, BMI and WC made the association with E-selectin disappear. Additional control for other potential confounders attenuated the association with TNF- $\alpha$. However, the associations with SAA and sVCAM-1 remained significant even after further control for dietary intakes; individuals in the highest quintile had higher levels of these inflammatory markers than those in the lowest quintile.

Findings from multiple linear regression models, with low- and high-fat dairy consumption as independent and log-transformed values of inflammatory markers as dependent variables, are shown in Table 3. After control for age, BMI, WC and other potential confounders, lowfat dairy consumption was inversely associated with CRP, IL-6 and sVCAM-1; with further adjustment for dietary intakes, the associations remained significant just for sVCAM-1. High-fat dairy intake was positively associated with log-transformed values of SAA and sVCAM-1, both before and after adjustment for all potential confounding variables. No overall significant associations were found between total dairy consumption, markers of inflammation and endothelial function. 
Table 1 General characteristics and dietary intakes of participants by quintiles of low- and high-fat dairy consumption*

\begin{tabular}{|c|c|c|c|c|c|c|c|c|c|c|c|c|c|c|}
\hline & \multicolumn{6}{|c|}{ Low-fat dairy consumption quintiles } & \multirow[b]{3}{*}{$P$ valuet } & \multicolumn{6}{|c|}{ High-fat dairy consumption quintiles } & \multirow[b]{3}{*}{$P$ valuet } \\
\hline & \multicolumn{2}{|c|}{1 (lowest) } & \multicolumn{2}{|c|}{3} & \multicolumn{2}{|c|}{5 (highest) } & & \multicolumn{2}{|c|}{1 (lowest) } & \multicolumn{2}{|c|}{3} & \multicolumn{2}{|c|}{5 (highest) } & \\
\hline & Mean & SD & Mean & SD & Mean & SD & & Mean & SD & Mean & SD & Mean & SD & \\
\hline Age (years) & 47 & 5 & 44 & 6 & 54 & 6 & $<0.01$ & 51 & 6 & 42 & 5 & 55 & 6 & $<0 \cdot 01$ \\
\hline BMI $\left(\mathrm{kg} / \mathrm{m}^{2}\right)$ & $28 \cdot 1$ & $3 \cdot 1$ & $27 \cdot 8$ & $3 \cdot 9$ & $26 \cdot 4$ & $3 \cdot 8$ & $<0.05$ & $27 \cdot 4$ & $3 \cdot 6$ & $28 \cdot 8$ & $3 \cdot 3$ & $27 \cdot 0$ & $3 \cdot 7$ & $<0.05$ \\
\hline Waist girth (cm) & 96 & 13 & 92 & 10 & 90 & 12 & $<0.05$ & 93 & 11 & 96 & 12 & 91 & 11 & $<0.05$ \\
\hline Physical activity (MET × h/week) & $13 \cdot 4$ & $11 \cdot 6$ & $12 \cdot 9$ & $9 \cdot 8$ & $17 \cdot 2$ & $11 \cdot 1$ & $<0.01$ & $15 \cdot 6$ & $10 \cdot 8$ & $14 \cdot 9$ & $9 \cdot 7$ & $15 \cdot 7$ & $11 \cdot 7$ & $0 \cdot 80$ \\
\hline Family history of diabetes (\%) & \multicolumn{2}{|c|}{9} & \multicolumn{2}{|c|}{9} & \multicolumn{2}{|c|}{11} & 0.39 & \multicolumn{2}{|c|}{7} & \multicolumn{2}{|c|}{12} & \multicolumn{2}{|c|}{9} & $0 \cdot 11$ \\
\hline Family history of stroke (\%) & \multirow{2}{*}{\multicolumn{2}{|c|}{$\begin{array}{l}0 \\
0\end{array}$}} & 2 & & \multicolumn{2}{|c|}{2} & 0.24 & 1 & & \multicolumn{2}{|c|}{2} & \multicolumn{2}{|l|}{1} & 0.87 \\
\hline Current daily smokers (\%) & & & 0 & & \multirow{2}{*}{\multicolumn{2}{|c|}{$\begin{array}{c}2 \\
28\end{array}$}} & $0 \cdot 11$ & \multirow{2}{*}{\multicolumn{2}{|c|}{$\begin{array}{c}1 \\
25\end{array}$}} & 2 & & \multirow{2}{*}{\multicolumn{2}{|c|}{$\begin{array}{c}0 \\
27\end{array}$}} & 0.52 \\
\hline Current oestrogen use (\%) & \multicolumn{2}{|c|}{19} & 3 & & & & $<0.05$ & & & 17 & & & & $<0.05$ \\
\hline \multicolumn{15}{|l|}{ Nutrients } \\
\hline Total energy (kcal/d) & 2316 & 29 & 2765 & 19 & 2477 & 22 & $<0.05$ & 2511 & 26 & 2589 & 23 & 2493 & 28 & $0 \cdot 67$ \\
\hline Carbohydrate ( $\%$ total energy) & 57 & 1 & 59 & 1 & 59 & 1 & $0 \cdot 18$ & 60 & 1 & 58 & 1 & 55 & 1 & $<0.01$ \\
\hline Protein (\% total energy) & $12 \cdot 7$ & 0.5 & $11 \cdot 8$ & 0.4 & $14 \cdot 1$ & 0.4 & $<0.05$ & $13 \cdot 1$ & 0.6 & $13 \cdot 6$ & 0.4 & $14 \cdot 3$ & 0.5 & 0.36 \\
\hline Fat ( $\%$ total energy) & $30 \cdot 1$ & $0 \cdot 7$ & $29 \cdot 4$ & 0.6 & $27 \cdot 0$ & $0 \cdot 8$ & $<0.05$ & $26 \cdot 6$ & 0.8 & $28 \cdot 2$ & 0.5 & $29 \cdot 9$ & $0 \cdot 6$ & $<0.05$ \\
\hline Cholesterol $(\mathrm{mg} / \mathrm{d})$ & 190 & 10 & 203 & 9 & 181 & 11 & 0.09 & 174 & 9 & 194 & 10 & 208 & 11 & $<0.05$ \\
\hline Dietary fibre $(\mathrm{g} / \mathrm{d})$ & 14 & 1 & 15 & 1 & 18 & 1 & $<0.05$ & 16 & 1 & 15 & 1 & 18 & 1 & 0.26 \\
\hline Vitamin $B_{2}(\mathrm{mg} / \mathrm{d})$ & 0.41 & 0.02 & 0.96 & 0.01 & $1 \cdot 61$ & 0.02 & $<0.01$ & 0.53 & 0.02 & $1 \cdot 02$ & 0.02 & $1 \cdot 56$ & $0 \cdot 01$ & $<0.01$ \\
\hline $\mathrm{Ma}(\mathrm{mg} / \mathrm{d})$ & 137 & 2 & 154 & 3 & 149 & 2 & $<0.05$ & 164 & 3 & 140 & 3 & 121 & 2 & $<0.05$ \\
\hline $\mathrm{Ca}(\mathrm{mg} / \mathrm{d})$ & 183 & 2 & 413 & 2 & 786 & 3 & $<0.01$ & 211 & 3 & 373 & 3 & 715 & 3 & $<0.01$ \\
\hline \multicolumn{15}{|l|}{ Foods $(\mathrm{g} / \mathrm{d})$} \\
\hline Fruit & 204 & 8 & 227 & 6 & 263 & 7 & $<0.01$ & 235 & 6 & 222 & 9 & 251 & 8 & $<0.05$ \\
\hline Vegetables & 186 & 6 & 178 & 5 & 194 & 5 & $<0.05$ & 182 & 5 & 189 & 6 & 192 & 6 & $0 \cdot 18$ \\
\hline Meat and fish & 85 & 3 & 97 & 3 & 91 & 2 & $<0.05$ & 90 & 3 & 88 & 3 & 94 & 3 & 0.39 \\
\hline Whole grains & 96 & 2 & 127 & 3 & 117 & 3 & $<0.05$ & 133 & 3 & 110 & 3 & 87 & 3 & $<0.05$ \\
\hline Refined grains & 205 & 4 & 192 & 9 & 209 & 6 & $0 \cdot 28$ & 217 & 7 & 201 & 8 & 184 & 6 & $<0.05$ \\
\hline Low-fat dairy & 33 & 2 & 88 & 2 & 152 & 3 & $<0.01$ & 112 & 3 & 73 & 2 & 86 & 3 & $<0.01$ \\
\hline High-fat dairy & 98 & 3 & 91 & 3 & 82 & 2 & $<0.05$ & 27 & 2 & 97 & 3 & 165 & 3 & $<0.01$ \\
\hline Hydrogenated fats & 37 & 1 & 24 & 1 & 17 & 1 & $<0.01$ & 12 & 1 & 20 & 1 & 43 & 1 & $<0.01$ \\
\hline Non-hydrogenated fats & 12 & 1 & 19 & 1 & 29 & 1 & $<0.01$ & 22 & 1 & 35 & 1 & 10 & 1 & $<0.01$ \\
\hline $\begin{array}{l}\text { MET, metabolic equivalent task. } \\
{ }^{*} \text { Data are mean and SD unless indicated. } \\
\text { tBy using ANOVA for continuous variable }\end{array}$ & nalysis of & ovarian & $r$ dietary & bles) & the $\chi^{2}$ te & $r$ cate & $\begin{array}{l}\text { intakes. } \\
\text { al variables }\end{array}$ & & & & & & & \\
\hline
\end{tabular}


Table 2 Multivariate-adjusted geometric means (SD) of circulating inflammatory marker levels across quintile categories of dairy consumption

\begin{tabular}{|c|c|c|c|c|c|c|c|c|c|c|c|c|c|c|}
\hline & \multicolumn{6}{|c|}{ Low-fat dairy consumption quintiles } & \multirow[b]{3}{*}{$P$ value ${ }^{*}$} & \multicolumn{6}{|c|}{ High-fat dairy consumption quintiles } & \multirow[b]{3}{*}{$P$ value } \\
\hline & \multicolumn{2}{|c|}{1 (lowest) } & \multicolumn{2}{|c|}{3} & \multicolumn{2}{|c|}{5 (highest) } & & \multicolumn{2}{|c|}{1 (lowest) } & \multicolumn{2}{|c|}{3} & \multicolumn{2}{|c|}{5 (highest) } & \\
\hline & Mean & SD & Mean & SD & Mean & SD & & Mean & SD & Mean & SD & Mean & SD & \\
\hline \multicolumn{15}{|l|}{ CRP (mg/l) } \\
\hline Crude & $2 \cdot 32$ & $2 \cdot 09$ & $1 \cdot 75$ & $2 \cdot 19$ & $1 \cdot 53$ & $1 \cdot 78$ & $<0.001$ & $1 \cdot 88$ & $1 \cdot 95$ & $2 \cdot 01$ & $2 \cdot 10$ & $1 \cdot 85$ & $2 \cdot 02$ & $0 \cdot 19$ \\
\hline Model It & $2 \cdot 08$ & 1.95 & $1 \cdot 71$ & $2 \cdot 03$ & $1 \cdot 61$ & $1 \cdot 70$ & $<0.01$ & $1 \cdot 90$ & $1 \cdot 91$ & 1.99 & $2 \cdot 05$ & $1 \cdot 82$ & 1.99 & 0.27 \\
\hline Model II & $1 \cdot 91$ & 1.93 & $1 \cdot 70$ & $2 \cdot 00$ & $1 \cdot 69$ & $1 \cdot 68$ & $<0.05$ & $1 \cdot 89$ & $1 \cdot 90$ & $1 \cdot 99$ & $2 \cdot 05$ & $1 \cdot 83$ & 1.98 & 0.29 \\
\hline Model III§ & $1 \cdot 78$ & $1 \cdot 89$ & $1 \cdot 72$ & 1.94 & $1 \cdot 81$ & $1 \cdot 63$ & $0 \cdot 14$ & $1 \cdot 87$ & $1 \cdot 88$ & $1 \cdot 98$ & $2 \cdot 04$ & $1 \cdot 83$ & $1 \cdot 98$ & 0.38 \\
\hline \multicolumn{15}{|l|}{ TNF- $\alpha$ (ng/l) } \\
\hline Crude & $5 \cdot 41$ & $2 \cdot 31$ & $4 \cdot 19$ & $1 \cdot 84$ & $3 \cdot 46$ & $1 \cdot 79$ & $<0.01$ & $4 \cdot 63$ & 1.90 & $4 \cdot 89$ & 1.97 & $4 \cdot 21$ & $1 \cdot 89$ & $<0.05$ \\
\hline Model I & $4 \cdot 28$ & $2 \cdot 14$ & 4.03 & $1 \cdot 81$ & $3 \cdot 50$ & 1.65 & $<0.05$ & 4.56 & $1 \cdot 88$ & $4 \cdot 70$ & 1.94 & $4 \cdot 35$ & $1 \cdot 86$ & $<0.05$ \\
\hline Model II & 3.93 & $2 \cdot 12$ & $3 \cdot 78$ & $1 \cdot 68$ & 3.56 & $1 \cdot 61$ & $<0.05$ & $4 \cdot 51$ & $1 \cdot 88$ & $4 \cdot 62$ & 1.93 & $4 \cdot 33$ & $1 \cdot 85$ & 0.09 \\
\hline Model III & $3 \cdot 54$ & $1 \cdot 88$ & $3 \cdot 69$ & $1 \cdot 57$ & $3 \cdot 64$ & $1 \cdot 58$ & 0.09 & $4 \cdot 46$ & $1 \cdot 85$ & $4 \cdot 54$ & $1 \cdot 91$ & $4 \cdot 38$ & $1 \cdot 85$ & 0.22 \\
\hline \multicolumn{15}{|l|}{ SAA (mg/l) } \\
\hline Crude & $5 \cdot 00$ & $2 \cdot 75$ & $4 \cdot 89$ & $3 \cdot 75$ & 4.95 & $3 \cdot 12$ & 0.67 & $4 \cdot 33$ & 3.02 & $5 \cdot 17$ & $3 \cdot 26$ & $5 \cdot 04$ & $3 \cdot 29$ & $<0.05$ \\
\hline Model I & $4 \cdot 84$ & $2 \cdot 67$ & 4.93 & $3 \cdot 66$ & 4.99 & 2.99 & 0.53 & $4 \cdot 25$ & 2.99 & $5 \cdot 06$ & $3 \cdot 16$ & $5 \cdot 11$ & $3 \cdot 25$ & $<0.01$ \\
\hline Model II & $4 \cdot 80$ & $2 \cdot 64$ & 4.90 & $3 \cdot 68$ & $5 \cdot 03$ & $2 \cdot 94$ & $0 \cdot 26$ & $4 \cdot 24$ & $2 \cdot 99$ & $5 \cdot 05$ & $3 \cdot 14$ & $5 \cdot 10$ & $3 \cdot 22$ & $<0.01$ \\
\hline Model III & $4 \cdot 78$ & $2 \cdot 61$ & $4 \cdot 88$ & $3 \cdot 62$ & $5 \cdot 01$ & $2 \cdot 92$ & $0 \cdot 21$ & $4 \cdot 22$ & $2 \cdot 96$ & $5 \cdot 01$ & $3 \cdot 14$ & $5 \cdot 12$ & $3 \cdot 20$ & $<0.01$ \\
\hline \multicolumn{15}{|l|}{ IL-6 (ng/l) } \\
\hline Crude & $2 \cdot 42$ & $1 \cdot 76$ & $2 \cdot 01$ & $2 \cdot 03$ & $1 \cdot 38$ & 1.94 & $<0.01$ & $2 \cdot 04$ & $1 \cdot 88$ & $2 \cdot 20$ & $2 \cdot 14$ & $1 \cdot 88$ & 1.90 & 0.20 \\
\hline Model I & 1.98 & $1 \cdot 68$ & 1.94 & 1.98 & $1 \cdot 66$ & $1 \cdot 89$ & $<0.01$ & 1.92 & $1 \cdot 80$ & $2 \cdot 05$ & $2 \cdot 10$ & $1 \cdot 91$ & $1 \cdot 88$ & 0.38 \\
\hline Model II & 1.91 & $1 \cdot 60$ & 1.95 & 1.94 & $1 \cdot 73$ & $1 \cdot 88$ & $<0.05$ & 1.90 & $1 \cdot 80$ & $2 \cdot 04$ & $2 \cdot 09$ & $1 \cdot 90$ & $1 \cdot 89$ & 0.43 \\
\hline Model III & $1 \cdot 84$ & 1.57 & 1.91 & 1.90 & $1 \cdot 79$ & $1 \cdot 85$ & $<0.05$ & $1 \cdot 88$ & $1 \cdot 82$ & $2 \cdot 02$ & $2 \cdot 08$ & 1.91 & $1 \cdot 87$ & 0.49 \\
\hline \multicolumn{15}{|c|}{ E-selectin (ng/l) } \\
\hline Crude & $56 \cdot 6$ & $17 \cdot 4$ & $50 \cdot 3$ & $18 \cdot 9$ & $47 \cdot 1$ & $21 \cdot 7$ & $<0.01$ & $49 \cdot 7$ & $19 \cdot 1$ & $52 \cdot 6$ & $19 \cdot 9$ & $50 \cdot 3$ & $18 \cdot 7$ & $<0.05$ \\
\hline Model I & $53 \cdot 4$ & $17 \cdot 6$ & $49 \cdot 1$ & $18 \cdot 1$ & $49 \cdot 5$ & $20 \cdot 4$ & $<0.05$ & $50 \cdot 1$ & $18 \cdot 8$ & $50 \cdot 8$ & $19 \cdot 3$ & $51 \cdot 5$ & $18 \cdot 9$ & $0 \cdot 18$ \\
\hline Model II & $51 \cdot 7$ & $17 \cdot 1$ & $49 \cdot 8$ & $17 \cdot 6$ & $49 \cdot 9$ & $19 \cdot 9$ & 0.08 & $50 \cdot 0$ & $18 \cdot 7$ & $50 \cdot 9$ & $19 \cdot 5$ & $51 \cdot 2$ & $18 \cdot 8$ & 0.26 \\
\hline Model III & $50 \cdot 5$ & $16 \cdot 8$ & $49 \cdot 4$ & $17 \cdot 5$ & $50 \cdot 3$ & $19 \cdot 6$ & $0 \cdot 21$ & $49 \cdot 3$ & $18 \cdot 6$ & $50 \cdot 5$ & $19 \cdot 8$ & $51 \cdot 9$ & $19 \cdot 1$ & 0.07 \\
\hline \multicolumn{15}{|c|}{ sICAM-1 $(\mu \mathrm{g} / \mathrm{l})$} \\
\hline Crude & 252 & 49 & 249 & 58 & 238 & 51 & $<0.05$ & 249 & 53 & 250 & 55 & 242 & 50 & $0 \cdot 14$ \\
\hline Model I & 248 & 48 & 247 & 56 & 243 & 50 & $0 \cdot 12$ & 251 & 51 & 246 & 54 & 246 & 51 & 0.23 \\
\hline Model II & 246 & 48 & 248 & 56 & 244 & 51 & 0.46 & 250 & 51 & 247 & 53 & 246 & 51 & 0.29 \\
\hline Model III & 242 & 47 & 247 & 55 & 247 & 50 & $0 \cdot 26$ & 248 & 50 & 244 & 51 & 248 & 50 & $0 \cdot 41$ \\
\hline \multicolumn{15}{|c|}{ sVCAM-1 $(\mu \mathrm{g} / \mathrm{l})$} \\
\hline Crude & 553 & 121 & 541 & 139 & 519 & 126 & $<0.01$ & 537 & 129 & 531 & 133 & 547 & 136 & $<0.05$ \\
\hline Model I & 545 & 119 & 537 & 137 & 526 & 125 & $<0.01$ & 530 & 127 & 525 & 131 & 555 & 134 & $<0.01$ \\
\hline Model II & 543 & 120 & 535 & 138 & 528 & 123 & $<0.01$ & 531 & 129 & 525 & 131 & 557 & 133 & $<0.01$ \\
\hline Model III & 540 & 120 & 531 & 138 & 531 & 123 & $<0.05$ & 529 & 128 & 523 & 130 & 557 & 135 & $<0.01$ \\
\hline
\end{tabular}

CRP, C-reactive protein; SAA, serum amyloid A; sICAM-1, soluble intercellular adhesion molecule-1; sVCAM, soluble vascular adhesion molecule-1.

*By using analysis of covariance.

tModel I: Adjusted for age, BMI and waist circumference. glucose, serum TAG concentrations, total cholesterol and HDL and LDL cholesterol.

§ Model II: Further adjusted for dietary variables including cholesterol intake, consumption of meats and fish, fruit and vegetables, whole and refined grains, hydrogenated and non-hydrogenated vegetable oils, percentage of energy from fat and mutual effects of high- and low-fat dairy intakes. 


\section{Discussion}

The current study, performed among women in Iran, found a significant inverse association of low-fat dairy consumption with sVCAM-1 and a positive association of high-fat dairy consumption with SAA and SVCAM-1. These associations persisted in multivariate models after known potential confounders were accounted for. Therefore, the relationships we reached are not likely to be attributed to other lifestyle variables associated with dairy consumption. To our knowledge, this is the first study reporting the epidemiological association between dairy consumption and circulating levels of inflammatory markers.

Although inflammation has recently obtained considerable attention as an important contributor to the development of many chronic diseases, little data are available relating food intakes to systemic inflammation. In the current study, the associations between dairy consumption and most inflammatory biomarkers were not significant; however, in some cases, such as SVCAM-1 (for both low- and high-fat dairy intakes) and SAA (just for high-fat dairy intake), we reached significant associations, even after control for dietary intakes. This finding is in accord with a recently reported cross-sectional study among a group of Spanish adults at high risk of CVD, in which a higher intake of dairy products was associated with lower concentrations of CRP and SICAM-1, even after adjustment for BMI ${ }^{(41)}$. However, findings from clinical trials indicated opposite results. Tricon et al. ${ }^{(42)}$, in a cross-over trial among healthy middle-aged men, showed that consumption of full-fat dairy products enriched with CLA did not affect inflammatory markers such as IL-6, sVCAM-1, sICAM-1, E-selectin or serum CRP concentrations. Compared to a diet with moderate dairy products, a hypoenergetic diet, high in dairy products, could not influence CRP levels among obese patients ${ }^{(43)}$. Supplementation with a milk drink for 12 weeks in hypertensive adults had no significant impact on inflammatory marker levels like CRP and IL- $6^{(44)}$. Not only dairy consumption $^{(40-43)}$, but also supplementation with $\mathrm{Ca}$ and vitamin $\mathrm{D}$ (two major components of dairy products) for 3 years could not affect systemic inflammation ${ }^{(35)}$. However, in two recent clinical trials ${ }^{(45,46)}$, vitamin D supplementation markedly reduced the serum levels of CRP and IL-6. In addition, low serum $25(\mathrm{OH})$ vitamin D concentrations have cross-sectionally been associated with high serum $\mathrm{CRP}^{(47)}$. It should be kept in mind that none of these trials has been designed to assess the effect of dairy consumption on inflammation, and most have reported such an effect as their accessory finding. Therefore, specifically designed clinical trials are required to assess dairy consumption's effect on systemic inflammation.

At this stage, no one can guess the mechanisms by which dairy consumption might affect systemic inflammation. However, findings from earlier studies might provide some clues. The Ca content of dairy

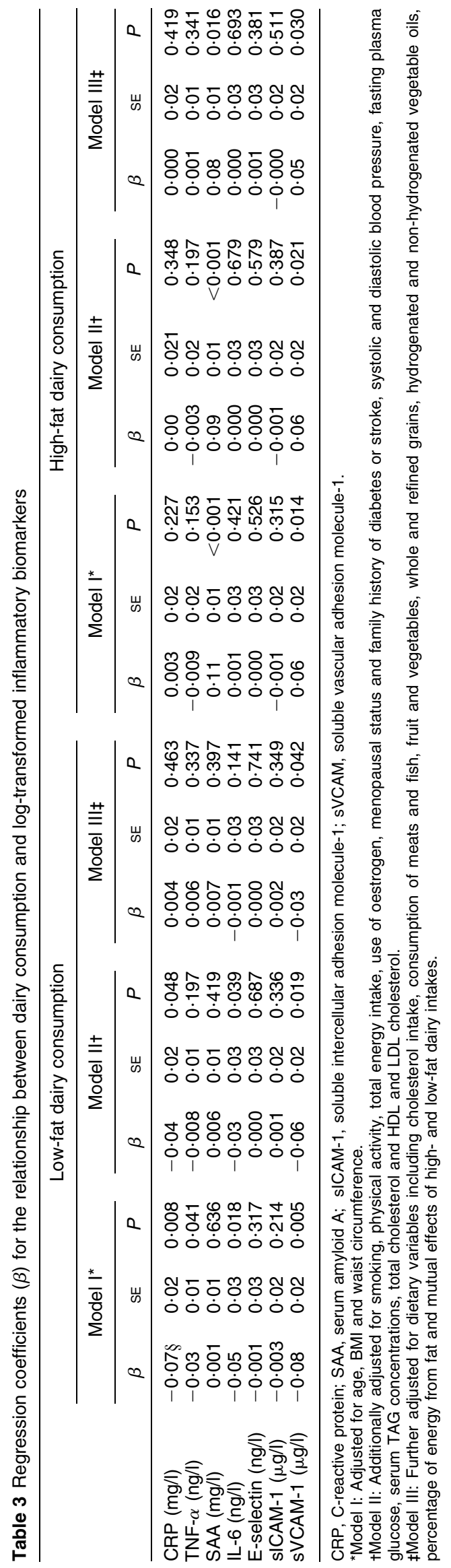


products has been reported to be responsible for dairy's favourable association with obesity and other metabolic abnormalities ${ }^{(26,31,34)}$; however, it does not seem to play a role in inflammation. Adjustment for $\mathrm{Ca}$ intake in a study by Salas-Salvado et al. ${ }^{(41)}$ could not explain the inverse association of dairy consumption with some inflammatory biomarkers. Our current analysis also indicated that total dairy intake had no significant associations with inflammation. Therefore, Ca does not seem to play a significantly role in the inflammation story. However, further studies are required to examine the relationship between $\mathrm{Ca}$ intake and systemic inflammation. Dairy's whey protein might be a contributing factor; however, milk drink supplemented with whey protein had no significant impact on inflammatory marker levels compared to a control dairy drink among hypertensive adults ${ }^{(44)}$. Also consumption of milk protein in conjunction with a high-fat meal did not acutely modify postprandial inflammation in young healthy men ${ }^{(37)}$. Besides Ca and protein, fat content of dairy might be an influencing factor for inflammation. In addition to saturated fats (SFA), dairy products contain CLA and trans fats (TFA). Some human and cell culture studies ${ }^{(36)}$ have suggested that CLA intake might attenuate the proinflammatory state. However, others indicated that daily consumption of $3 \mathrm{~g}$ of different isomers of CLA did not affect plasma CRP levels ${ }^{(48,49)}$. TFA intake has been related to elevated levels of inflammatory biomarkers ${ }^{(14,50)}$. Significant positive association between high-fat dairy intake and some inflammatory biomarkers in the current study could be attributed to SFA and TFA content of these products. The hypothesis that TFA from dairy products might affect human health differently from those in hydrogenated fats ${ }^{(51)}$ should also be kept in mind. Further studies are needed to examine this hypothesis. Other components of dairy products, such as vitamin $\mathrm{B}_{2}$ and bioactive compounds, have not yet been examined for their effects on systemic inflammation.

\section{Conclusion}

Several limitations need to be considered in the interpretation of our findings. The major concern is the crosssectional design of the study that does not allow us to infer causal relationships. However, the appropriate analysis of cross-sectional data represents a valuable initial step in identifying relationships between diet and disease. Possible misclassifications of participants due to the use of an FFQ for assessing dietary intakes should also be considered. This is particularly relevant for the inclusion of cheese and other dairy products in the same category, because each $100 \mathrm{~g}$ of Iranian cheese gives very seldom less than $30 \mathrm{~g}$ of fat, most often much more, while other products in the high-fat dairy category, such as yoghurt, give almost $6-7 \mathrm{~g}$ fat/100 g. Unfortunately, our database did not include fat intake contributed from dairy products and it just had a variable on total fat intake. We tried to control for known lifestyle variables associated with dairy consumption; however, residual confounding (like lack of data on family history of stroke and heart diseases) in our study, as in all epidemiological studies, is inevitable. We used a single blood measurement of inflammation, but that may not accurately reflect longterm inflammatory status. Lack of control for glycaemic load, a dietary agent that has been shown to correlate with inflammation ${ }^{(52)}$, should also be kept in mind. However, the associations we observed are unlikely to be confounded significantly by dietary glycaemic load, because the extensive adjustments we made had minimal impacts on correlations.

Given the aforementioned limitations, we have found evidence indicating an independent relationship between dairy consumption and some markers of inflammation and endothelial dysfunction. Further studies are required to identify responsible components of dairy products and related mechanisms of action.

\section{References}

1. Berg AH \& Scherer PE (2005) Adipose tissue, inflammation, and cardiovascular disease. Circ Res 96, 939-949.

2. Williams KJ \& Tabas I (2002) Atherosclerosis and inflammation. Science 297, 521-522.

3. Libby P (2006) Inflammation and cardiovascular disease mechanisms. Am J Clin Nutr 83, 456S-460S.

4. Pickup JC (2004) Inflammation and activated innate immunity in the pathogenesis of type 2 diabetes. Diabetes Care 27, 813-823.

5. Dandona P, Aljada A, Chaudhuri A et al. (2005) Metabolic syndrome: a comprehensive perspective based on interactions between obesity, diabetes and inflammation. Circulation 111, 1448-1454.

6. No authors listed (2007) Simple changes in diet can protect you against friendly fire. What you eat can fuel or cool inflammation, a key driver of heart disease, diabetes, and other chronic conditions. Harv Heart Lett 17, 3.

7. Ridker PM, Rifai N, Stampfer MJ et al. (2000) Plasma concentration of interleukin- 6 and the risk of future myocardial infarction among apparently healthy men. Circulation 101, 1767-1772.

8. Ridker PM, Rifai N, Pfeffer M et al. (2000) Elevation of tumor necrosis factor-alpha and increased risk of recurrent coronary events after myocardial infarction. Circulation 101, 2149-2153.

9. Esposito K \& Giugliano D (2004) The metabolic syndrome and inflammation: association or causation? Nutr Metab Cardiovasc Dis 14, 228-232.

10. Esmaillzadeh A \& Azadbakht L (2008) Consumption of hydrogenated versus nonhydrogenated vegetable oils and risk of insulin resistance and the metabolic syndrome among Iranian adult women. Diabetes Care 31, 223-226.

11. Frohlich M, Sund M, Lowel $\mathrm{H}$ et al. (2003) Independent association of various smoking characteristics with markers of systemic inflammation in men. Results from a representative sample of the general population (MONICA Augsburg Survey 1994/95). Eur Heart J 24, 1365-1372.

12. Stokes KY, Cooper D, Tailor A et al. (2002) Hypercholesterolemia promotes inflammation and microvascular dysfunction: 
role of nitric oxide and superoxide. Free Radic Biol Med 33, 1026-1036.

13. Hjelstuen A, Anderssen SA, Holme I et al. (2006) Markers of inflammation are inversely related to physical activity and fitness in sedentary men with treated hypertension. Am J Hypertens 19, 669-675.

14. Mozaffarian D, Pischon T, Hankinson SE et al. (2004) Dietary intake of trans fatty acids and systemic inflammation in women. Am J Clin Nutr 79, 606-612.

15. Pischon T, Hankinson SE, Hotamisligil GS et al. (2003) Habitual dietary intake of $n-3$ and $n-6$ fatty acids in relation to inflammatory markers among US men and women. Circulation 108, 155-160.

16. King DE, Egan BM, Woolson RF et al. (2007) Effect of a high-fiber diet vs. a fiber-supplemented diet on C-reactive protein level. Arch Intern Med 167, 502-506.

17. Bo S, Durazzo M, Guidi S et al. (2006) Dietary magnesium and fiber intakes and inflammatory and metabolic indicators in middle-aged subjects from a population-based cohort. Am J Clin Nutr 84, 1062-1069.

18. Esmaillzadeh A, Kimiagar M, Mehrabi Y et al. (2007) Dietary patterns and markers of systemic inflammation among Iranian women. J Nutr 137, 992-998.

19. Nettleton JA, Steffen LM, Mayer-Davis EJ et al. (2006) Dietary patterns are associated with biochemical markers of inflammation and endothelial activation in the multi-ethnic study of atherosclerosis (MESA). Am J Clin Nutr 83, 1369-1379.

20. Esposito K, Ciotola M \& Giugliano D (2006) Mediterranean diet, endothelial function and vascular inflammatory markers. Public Health Nutr 9, 1073-1076.

21. Esmaillzadeh A \& Azadbakht L (2008) Home use of vegetable oils, markers of systemic inflammation and endothelial dysfunction among women. Am J Clin Nutr 88, 913-921.

22. Azadbakht L \& Esmaillzadeh A (2009) Red meat intake is associated with metabolic syndrome and plasma C-reactive protein concentration in women. J Nutr 139, 335-339.

23. Wannamethee SG, Lowe GD, Rumley A et al. (2006) Associations of vitamin C status, fruit and vegetable intakes, and markers of inflammation and hemostasis. Am J Clin Nutr 83, 567-574.

24. Esmaillzadeh A, Kimiagar M, Mehrabi Y et al. (2006) Fruit and vegetable intakes, C-reactive protein, and the metabolic syndrome. Am J Clin Nutr 84, 1489-1497.

25. Azadbakht L, Kimiagar M, Mehrabi Y et al. (2007) Soy consumption, markers of inflammation, and endothelial function: a cross-over study in postmenopausal women with the metabolic syndrome. Diabetes Care 30, 967-973.

26. Zemel MB (2005) The role of dairy foods in weight management. J Am Coll Nutr 24, 537S-546S.

27. Rosell M, Hakansson NN \& Wolk A (2006) Association between dairy food consumption and weight change over 9 y in 19,352 perimenopausal women. Am J Clin Nutr 84, 1481-1488.

28. Mirmiran P, Esmaillzadeh A \& Azizi F (2005) Dairy consumption and body mass index: an inverse relationship. Int J Obes 29, 115-121.

29. Liu S, Choi HK, Ford E et al. (2006) A prospective study of dairy intake and the risk of type 2 diabetes in women. Diabetes Care 29, 1579-1584.

30. Choi HK, Willett WC, Stampfer MJ et al. (2005) Dairy consumption and risk of type 2 diabetes mellitus in men: a prospective study. Arch Intern Med 165, 997-1003.

31. Azadbakht L, Mirmiran P, Esmaillzadeh A et al. (2005) Dairy consumption is inversely associated with the prevalence of the metabolic syndrome in Tehranian adults. Am J Clin Nutr 82, 523-530.

32. Pfeuffer M \& Schrezenmeir J (2007) Milk and the metabolic syndrome. Obes Rev 8, 109-118.

33. Pereira MA, Jacobs DR Jr, Van Horn L et al. (2002) Dairy consumption, obesity, and the insulin resistance syndrome in young adults: the CARDIA study. JAMA $\mathbf{2 8 7}$, 2081-2089.

34. Zemel MB, Richards J, Milstead A et al. (2005) Effects of calcium and dairy on body composition and weight loss in African-American adults. Obes Res 13, 1218-1225.

35. Pittas AG, Harris SS, Stark PC et al. (2007) The effects of calcium and vitamin D supplementation on blood glucose and markers of inflammation in nondiabetic adults. Diabetes Care 30, 980-986.

36. Moloney F, Toomey S, Noone E et al. (2007) Antidiabetic effects of cis-9, trans-11-conjugated linoleic acid may be mediated via anti-inflammatory effects in white adipose tissue. Diabetes 56, 574-582.

37. Campbell CG, Brown BD, Dufner D et al. (2006) Effects of soy or milk protein during a high-fat feeding challenge on oxidative stress, inflammation, and lipids in healthy men. Lipids 41, 257-265.

38. Esmaillzadeh A \& Azadbakht L (2008) Major dietary patterns in relation to general obesity and central adiposity among Iranian women. J Nutr 138, 358-363.

39. Esmaillzadeh A, Kimiagar M, Mehrabi Y et al. (2007) Dietary patterns, insulin resistance, and prevalence of the metabolic syndrome in women. Am J Clin Nutr 85, 910-918.

40. Esmaillzadeh A \& Azadbakht L (2008) Food intake patterns may explain the high prevalence of cardiovascular risk factors among Iranian women. J Nutr 138, 1469-1475.

41. Salas-Salvado J, Garcia-Arellano A, Estruch R et al. (2008) Components of the Mediterranean-type food pattern and serum inflammatory markers among patients at high risk for cardiovascular disease. Eur J Clin Nutr 62, 651-659.

42. Tricon S, Burdge GC, Jones EL et al. (2006) Effects of dairy products naturally enriched with cis- 9 , trans- 11 conjugated linoleic acid on the blood lipid profile in healthy middleaged men. Am J Clin Nutr 83, 744-753.

43. Thompson WG, Rostad Holdman N, Janzow DJ et al. (2005) Effect of energy-reduced diets high in dairy products and fiber on weight loss in obese adults. Obes Res 13, 1344-1353.

44. Lee YM, Skurk T, Hennig M et al. (2007) Effect of a milk drink supplemented with whey peptides on blood pressure in patients with mild hypertension. Eur J Nutr 46, 21-27.

45. Van den Berghe G, Van Roosbroeck D, Vanhove P et al. (2003) Bone turnover in prolonged critical illness: effect of vitamin D. J Clin Endocrinol Metab 88, 4623-4632.

46. Timms PM, Mannan N, Hitman GA et al. (2002) Circulating MMP9, vitamin D and variation in the TIMP-1 response with VDR genotype: mechanisms for inflammatory damage in chronic disorders? QJM 95, 787-796.

47. Cigolini M, Iagulli MP, Miconi V et al. (2006) Serum 25-hydroxyvitamin D3 concentrations and prevalence of cardiovascular disease among type 2 diabetic patients. Diabetes Care 29, 722-724.

48. Ramakers JD, Plat J, Sébédio JL et al. (2005) Effects of the individual isomers cis-9, trans-11 vs. trans-10,cis-12 of conjugated linoleic acid (CLA) on inflammation parameters in moderately overweight subjects with LDL-phenotype B. Lipids 40, 909-918.

49. Bhattacharya A, Banu J, Rahman M et al. (2006) Biological effects of conjugated linoleic acids in health and disease. J Nutr Biochem 17, 789-810.

50. Lopez-Garcia E, Schulze MB, Meigs JB et al. (2005) Consumption of trans fatty acids is related to plasma biomarkers of inflammation and endothelial dysfunction. J Nutr 135, 562-566.

51. Gebauer SK, Psota TL \& Kris-Etherton PM (2007) The diversity of health effects of individual trans fatty acid isomers. Lipids $\mathbf{4 2}, 787-799$.

52. Liu S, Manson JE, Buring JE et al. (2002) Relation between a diet with a high glycemic load and plasma concentrations of high-sensitivity C-reactive protein in middle-aged women. Am J Clin Nutr 75, 492-498. 